\title{
BMJ Open Epidemiology of at-risk alcohol use and associated comorbidities of interest among community-dwelling older adults: a protocol for a systematic review
}

\author{
Maria Latanioti, ${ }^{1}$ Jean-Pierre Schuster, ${ }^{1}$ Joelle Rosselet Amoussou, ${ }^{2}$ \\ Marie-Pierre F. Strippoli, ${ }^{3}$ Armin von Gunten, ${ }^{4}$ Karsten Ebbing, ${ }^{1}$ Henk Verloo
}

To cite: Latanioti M, Schuster J$\mathrm{P}$, Rosselet Amoussou J, et al. Epidemiology of at-risk alcohol use and associated comorbidities of interest among community-dwelling older adults: a protocol for a systematic review. BMJ Open 2020;10:e035481. doi:10.1136/ bmjopen-2019-035481

- Prepublication history and additional material for this paper are available online. To view these files, please visit the journal online (http://dx.doi. org/10.1136/bmjopen-2019035481).

Received 04 November 2019 Revised 28 November 2019 Accepted 04 December 2019

\section{Check for updates}

(C) Author(s) (or their employer(s)) 2020. Re-use permitted under CC BY-NC. No commercial re-use. See rights and permissions. Published by BMJ.

For numbered affiliations see end of article.

Correspondence to Professor Henk Verloo; henk.verloo@hevs.ch

\section{ABSTRACT}

Introduction There is little epidemiological evidence and knowledge about at-risk alcohol use among communitydwelling older adults and their chronic and acute alcoholrelated comorbidities of interest. This systematic review will summarise and examine relevant studies about the epidemiology of at-risk alcohol use and associated comorbidities of interest in this population.

Methods We will search the following databases, without language or date restrictions, from inception to 31 August 2019: Embase.com, Medline Ovid SP, Pubmed (NOT medline[sb]), CINAHL EBSCO, PsycINFO Ovid SP, Central-Cochrane Library Wiley and Web of Science (Core Collection). Search strategies will be developed in collaboration with a librarian. We will use predefined search terms for alcoholism, epidemiology, the elderly, living place and comorbidities of interest, as well as terms related to the identification of "measurements", "tools" or "instruments" for measuring harm from alcohol use. At-risk status will be determined by the amount of alcohol consumed and any comorbidities of interest associated with at-risk alcohol use, with the latter being documented separately or using an assessment tool for at-risk drinking. We will also examine the bibliographies of all the relevant articles found and search for unpublished studies. We will consider publications in all languages.

Ethics and dissemination No ethical approval is necessary. Results will be presented in national and international conferences on addiction and published in a peer-reviewed journal.

PROSPERO registration number CRD42018099965.

\section{INTRODUCTION}

Alcohol consumption and misuse is a major substance abuse problem among communitydwelling older adults. ${ }^{1-3}$ Excessive alcohol use is a well-known health risk among elderly people. ${ }^{4}$ It is widely documented that older adults' responses to alcohol are different from those of younger adults due to the physiological process of ageing. ${ }^{56}$ The physiological changes occurring with ageing, as well as differences in the activities and responsibilities of older people, are used for establishing
Strengths and limitations of this study

- Will provide specific, synthesised knowledge about the epidemiology of at-risk alcohol use among older and very old community-dwelling older adults.

- Will include a selection of associated comorbidities of interest (cognitive impairment, diabetes, obesity, heart failure, depression, hypertension, insomnia, liver failure, pain and anxiety) related to at-risk drinking among old and very old communitydwelling older adults.

- Will report on the different definitions of at-risk drinking among old and very old communitydwelling adults in different studies and countries, including the quantification of their consumption.

- Will use inclusion criteria which impose no restrictions on language, study age or geographic location.

- Nevertheless, one potential limitation could be the introduction of bias due to the authors' personal judgements in their assessments of the studies included.

the criteria of alcoholism and ageing. ${ }^{78}$ Older and very old adults are more vulnerable to the effects of alcohol because of metabolic and other changes in their bodies and their high rate of chronic diseases. ${ }^{17}$

Epidemiological studies have shown declining alcohol use with age. ${ }^{49}$ However, the number of older adults exhibiting at-risk drinking is likely to increase when the age cohort born after 1950 (the 'baby boomers'), with its heavier drinking habits, reaches old age. ${ }^{1011}$ The sociodemographic and political changes affecting this generation, especially in western countries, had a great impact on the way people used and abused alcohol and psychotropic substances. Individual differences, like general health, physical or psychiatric comorbidities, drinking-age onset and the presence of cognitive impairment can alter responses to alcohol among older adults. ${ }^{612}$ Adults reaching very old age 
are more likely to suffer from cognitive decline, and the typical adverse effects of heavy alcohol consumption may worsen. ${ }^{1314}$ Dementia resulting from alcoholism is often diagnosed in older adults when their evident cognitive and functional decline cannot be attributed to a progressive neurodegenerative disorders like Alzheimer's disease, or when their clinical history reveals chronic and severe drinking. Adults reaching very old age also undergo the systemic physiological and neural changes that may make them more susceptible to the effects of alcohol. ${ }^{1516}$

Additionally, due to the metabolic and neurological changes that occur with at-risk drinking in old age, alcohol consumption is one of the lifestyle issues which should be considered in cases involving diabetic, hypertensive and depressive older patients. ${ }^{17}$ Many pharmacological treatments have potential interactions with alcohol. ${ }^{18}$ Unfortunately, the criteria for alcohol abuse and dependence established by the DSM or ICD (Diagnostic and Statistical Manual of Mental Disorders or International Classification of Diseases) manuals are not adapted for older and very old adults. ${ }^{19}{ }^{20}$ Bearing this in mind, physicians often use the at-risk, moderate and heavy drinking model to characterise drinking patterns more effectively. ${ }^{21}{ }^{22}$ Studies have recommended that at-risk drinking should be considered on a case by case basis. ${ }^{23}$ Fundamentally, we know that alcohol consumption in older age can compromise general health. ${ }^{512}$ Nonetheless, defining $a t$ risk drinking has shown itself to be methodologically and conceptually challenging. ${ }^{24}{ }^{25}$ Factors such as drinking volumes, drinking patterns, types of drinks and drink size have been considered in efforts to define a threshold for low-risk alcohol use. ${ }^{326}$ Limits vary between countries and even between regions in the same country (eg, Spain). ${ }^{2627}$ At-risk drinking can be defined as alcohol consumption beyond the limits that can lead to all-cause mortality, chronic conditions and acute consequences. ${ }^{61528}$

The American National Institute on Alcohol Abuse and Alcoholism's definition of low-risk alcohol consumptionthe lowest threshold ${ }^{29}$-has established criteria for lowrisk drinking for adults over 65 years old. It recommends a pattern involving no more than one alcoholic drink per day and sets a standard amount of drink: one can (12 US fl oz or approx. $355 \mathrm{~mL}$ ) of beer, one glass (5 US fl oz or approx. $148 \mathrm{~mL}$ ) of wine, a small glass (4 US $\mathrm{fl} \mathrm{oz}$ or approx. $118 \mathrm{~mL}$ ) of liquor or one shot (1.5 US $\mathrm{fl} \mathrm{oz} \mathrm{or} \mathrm{approx.} 44 \mathrm{~mL}$ ) of hard liquor. Translated into equivalent measures of pure alcohol, as documented in the literature, these equate to 0.6 fluid ounces in imperial measurements and $\sim 17 \mathrm{~g}$ in metric measurements. ${ }^{26}$ Multiple features of at-risk drinking are documented in the literature. ${ }^{25}$ However, the USA's low-risk drinking guidelines are generally in line with the risk levels observed in the scientific literature. ${ }^{230}$ Comorbidity is defined as the presence of more than one distinct medical condition in an individual. This condition can exist simultaneously with, but independently of, another condition, or it can be related. ${ }^{31}{ }^{32}$ The comorbidities of interest to at-risk alcohol use among older adults are hypertension, depression, pain, liver disease, insomnia, cognitive deficiency, diabetes and anxiety. Our systematic review will explore at-risk alcohol use because we found no reviews on this issue in the international literature, even though awareness of at-risk alcohol use among the elderly is rising among the general population and healthcare professionals. This systematic review will only examine at-risk alcohol (and not other substances) consumption because it is the substance for which we have the most information. The following research questions will guide this systematic review:

- What is the reported epidemiology of alcohol consumption, age of onset and severity of use among home-dwelling older adults?

- What are the psychiatric and somatic comorbidities occurring in this population?

- Which tools and measurements are used to document the comorbidities associated with at-risk drinking?

- Do we have epidemiological data concerning alcohol use among very old adults?

- Is there an association between drinking volume and alcohol-related harm?

\section{METHODS}

This review will be conducted following the recommendations and harms-reporting checklist of the Preferred Reporting Items for Systematic Review and Meta-Analysis Protocols, ${ }^{334}$ the reporting proposals of the Meta-analysis Of Observational Studies in Epidemiology (MOOSE) ${ }^{35}$ and the methods outlined in the Cochrane Handbook for Systematic Reviews of Interventions ${ }^{36}$ (online supplementary 1).

Database searches and searches using other techniques will be completed in September 2019. Retrieved articles will then be screened. The entire study is expected to be completed by September 2020 .

\section{Inclusion criteria}

Types of studies

This review will include retrospective and prospective epidemiological studies, cohort studies, case-control studies, controlled before-and-after studies, interruptedtime-series studies and controlled trials with inappropriate randomisation (quasiexperimental studies) ${ }^{3738} \mathrm{We}$ will put no language restrictions on our search for papers.

\section{TYPES OF PARTICIPANTS}

This review will consider studies involving home-dwelling adults with a minimum mean age of 60 years old, as well as studies with participants aged 55 years old or more who consume at least one alcoholic drink per day. Although various definitions of old-age exist, we will refer to the United Nations cut-off considering anybody aged 60 or more to be an older person. ${ }^{39}$ 


\section{Types of outcome measures}

To highlight the epidemiology of at-risk alcohol consumption and the presence of comorbidities of interest in home-dwelling older and very old adults, this systematic review protocol will use the drinking limit established by America's National Institute on Alcohol Abuse and Alcoholism. ${ }^{40}{ }^{41}$ Considering at-risk drinking as a medical condition, we have chosen to search for epidemiological data concerning medical conditions simultaneously present among older adults with at-risk drinking behaviours. ${ }^{30} 40$ We have chosen not to limit our search to medical conditions cited in the Comorbidity Alcohol Risk Evaluation Tool. ${ }^{42}$ The following conditions are not captured by this tool: osteoporosis, behavioural disorders, other drug use, social isolation, Oto-rhino-laryngology (ORL) cancers, and falls and trauma.

In addition, we will cross-reference at-risk alcohol consumption tools involving medical conditions with those referenced in the CoLausPsyCoLaus study of the general population of Lausanne, Switzerland. ${ }^{43-45}$ This choice was made purposefully as, in the future, we plan to analyse Swiss data to compare them with data found in the international literature.

- The review's primary outcome measures will be the:

- Epidemiology of at-risk alcohol consumption, age of onset and severity of alcohol use (amount, frequency and types of drinks).

- The review's secondary outcome measures will be the:

- Psychiatric and somatic comorbidities frequently occurring among home-dwelling older adults with at-risk alcohol consumption.

- Documentation of the tools and measurements of comorbidities associated with at-risk drinking.

- Presence of epidemiological data on very old adults' drinking habits.

- Associations between drinking volume and alcoholrelated harm.

\section{Search methods for the identification of relevant studies} Electronic searches

We will search the following databases, with no language or date restrictions: Embase.com, Medline Ovid SP, Pubmed (NOT medline[sb]), CINAHL EBSCO, PsycINFO Ovid SP, Central-Cochrane Library Wiley and Web of Science (Core Collection).

\section{Hand and grey literature searches}

We will search the reference lists of the relevant articles identified for unpublished studies (grey literature) and for experts in the field who could be contacted.

The search strategies will be adapted to each database's syntax and subject headings. Descriptor terms will include:

- Terms for alcoholism: "at-risk alcohol use", "heavy drinking", "binge drinking", "alcohol dependence", "alcohol abuse", "Wernicke syndrome", "Korsakoff dementia", "at-risk drinking", "alcohol consumption", "alcohol dependence" and "geriatric drink*".
- Epidemiological terms: "epidemiology", "occurrence", "prevalence", "incidence" and "occasionally".

- Terms for elderly: "home-dwelling older adults", "elderly", "aged", "home-care patients", "older adults" and "very old adults".

- Terms for living place: "home-dwelling", "living in place", "homebound", "primary care", "community health services", "community hospital", "ambulatory care", "outpatient clinics", "hospital”, "ambulatory care facilities", "day-care", "primary healthcare", "community health centres", "health services for the aged", "community", "domiciliary", "home or homecare or home-based", "outpatient", "day patient", "community care", "home-care services", "general practice" and "urban population".

- Terms related to the comorbidities of interest: "cognitive impairment", "diabetes", "obesity", "heart failure”, "depression", "hypertension", "insomnia", "liver failure", "pain", "dementia", "cognitive deficiency" and "anxiety".

- Terms related to the identification of "measurements", "tools" and "instruments" for measuring the harm of alcohol use.

Terms related to the identification of "measurements", "tools" and "instruments" for measuring the harm of alcohol use.

Online supplementary 2 presents the search strategy and equations.

\section{Data collection and analysis}

Study selection

Three reviewers-ML, KE and J-PS-will independently screen the titles and abstracts identified in the searches to assess which studies meet the inclusion criteria. Disagreements will be resolved through discussion or, if needed, a consensus will be reached after discussion with the coauthors (HV, AV-G).

The reviewers will then independently assess the fulltext articles to ensure that they meet the inclusion criteria. Disagreements will be discussed and resolved with the coauthors (HV, AV-G). A flowchart of the trial selection process has been drawn in accordance with the Preferred Reporting Items for Systematic Review and Meta-Analysis Protocols (PRISMA) statement ${ }^{46}$ (online supplementary 3).

\section{Data extraction}

Three authors-ML, KE and HV-will extract the data independently using a specially designed, standardised data extraction form (online supplementary 4). Discrepancies will be resolved through discussion and consultation with the coauthor (AV-G).

The following information will be extracted from each study included: (1) study authors, year of publication and country where the study was conducted; (2) study characteristics (including setting and design, duration of follow-up and sample size); (3) participants' characteristics (including age, sex, social status, marital status, 
educational status, activity, age of onset of alcohol consumption, level of autonomy, history of violence); (4) comorbidities of interest (hypertension, depression, pain, liver disease, insomnia, cognitive deficiency, diabetes, anxiety); (5) types of outcome measures.

\section{Assessment of the risks of bias in included studies}

Three reviewers-ML, KE and J-PS—will independently assess the risks of bias in all the retrospective and prospective epidemiological studies, cohort studies, case-control studies, controlled before-and-after studies, interruptedtime-series studies and controlled trials with inappropriate randomisation (quasiexperimental studies) included. Disagreements will be resolved through discussion and consultation with the coauthors (HV, AV-G).

We will use the validated Robins-I tool for assessing the risk of bias in non-randomised studies of interventions (NRSI). ${ }^{47}$ This tool covers two dimensions and seven domains through which bias might be introduced into an NRSI: (i) preintervention and at intervention (bias due to confounding, bias in the selection of study participants and bias in the classification of the intervention) and (ii) postintervention (bias due to deviations from intended interventions, bias due to missing data, bias in the measurement of outcomes and bias in the selection of the reported result). ${ }^{47}$ Any disagreements in quality assessments will be resolved through discussion.

Our search strategy will be very careful to select original research papers only and will try to avoid duplicates of published data of longitudinal studies. Additionally, our extraction sheet will pay special attention to longitudinal cohort studies and secondary analyses of published results.

\section{Statistical analyses}

Statistical analyses will be conducted following the recommendations of the Cochrane Handbook for Systematic Reviews of Interventions ${ }^{48}$ and the PRISMA and MOOSE statements. $^{33}$

For dichotomous outcomes, average intervention effects will be calculated as relative risks with $95 \%$ CIs, using a random effects model. ${ }^{49}$ For continuous data, a random effects model will be used to calculate weighted mean differences with $95 \%$ CIs. If required, we will calculate SD from the SE or $95 \%$ CIs presented in the articles. Heterogeneity will be quantified using the $\mathrm{I}^{2}$ and $\chi^{2}$ tests. Funnel plots will be drawn, and Egger tests will be computed to explore the possibility of publication bias. ${ }^{50}$ Reasons for heterogeneity in effect estimates will be sought in meta-analyses. ${ }^{51}$ To explore the possible determinants of heterogeneity, we will conduct subgroup analyses according to selected study characteristics (eg, participants' ages, country where the study was conducted, amounts of alcohol). Furthermore, sensitivity analyses will be conducted by: (1) excluding relatively small studies (with fewer than 20 participants per randomisation group); (2) restricting the analyses to studies of good quality. Data will be analysed using SPSS software (V.25.0) and Review Manager V.5.3. ${ }^{2}$

\section{Patient and public involvement}

No patients or members of the public were involved in the preparation of this protocol for a systematic review.

\section{DISCUSSION}

At-risk alcohol use among older adults is a common health problem. It is under-diagnosed by primary care physicians, partially due to the lack of up-to-date epidemiological data ${ }^{21}$ and partially due to the lack of relevant and specific diagnostic tools and instruments. ${ }^{2452}$ We will propose recommendations about screening tools and instruments which might be particularly appropriate for clinicians to use when screening for alcohol misuse in certain contexts.

Before being able to highlight the lack of data on alcohol consumption among older and very old adults or trying to establish relevant diagnostic criteria and assessment methods, it is important to find out about the existing epidemiological data at an international level. It is equally important to acknowledge the difficulty in defining at-risk drinking at an international level and the methods used to extract this data. ${ }^{2}$

Demonstrating a high prevalence, frequency or incidence of at-risk alcohol use among older home-dwelling adults could encourage physicians to use existing screening tests. This could be an important measure, considering that alcohol-related health problems reduce the length and quality of life. ${ }^{53}$ Recent studies, however, have demonstrated that elevated alcohol consumption cannot be evaluated solely in terms of frequency ${ }^{54}$; it is also necessary to know the types of drinks ingested. ${ }^{55}$

\section{Ethics and dissemination}

No ethical clearance is necessary. We expect to complete the study in September 2020. Results will be presented at national and international conferences on addiction and published in an international peer-reviewed journal.

\section{Author affiliations}

${ }^{1}$ Department of Psychiatry, Lausanne University Hospital, Service of Old Age Psychiatry, Prilly, Switzerland

${ }^{2}$ Department of Psychiatry, Lausanne University Hospital, Education and Research Department, University of Lausanne, Prilly, Switzerland

${ }^{3}$ University of Lausanne, Centre for Psychiatric Epidemiology and Psychopathology (CEPP), Lausanne, Switzerland

${ }^{4}$ Department of Psychiatry Lausanne University Hospital, Service of Old Age Psychiatry, Prilly, Switzerland

${ }^{5}$ Nursing Sciences, School of Health Sciences HES-SO Valais/Wallis, Sion, Switzerland

${ }^{6}$ Department of Psychiatry, Lausanne University Hospital, Service of Old Age Psychiatry, Prilly, Switzerland

Contributors $\mathrm{ML}$ is the guarantor and all the authors contributed to drafting the protocol. All authors contributed to the development of the selection criteria, data extraction and analysis, and the search strategy. J-PS, HV, KE and AV-G provided expertise on evidence-based practice. All the authors approved the final protocol manuscript. 
Funding This research received a grant from the public University 'School of Health Sciences, HES-SO Valais/Wallis, CH-1950 Sion, Switzerland'.

Competing interests None declared.

Patient consent for publication Not required

Provenance and peer review Not commissioned; externally peer reviewed.

Open access This is an open access article distributed in accordance with the Creative Commons Attribution Non Commercial (CC BY-NC 4.0) license, which permits others to distribute, remix, adapt, build upon this work non-commercially, and license their derivative works on different terms, provided the original work is properly cited, appropriate credit is given, any changes made indicated, and the use is non-commercial. See: http://creativecommons.org/licenses/by-nc/4.0/.

\section{ORCID iD}

Henk Verloo http://orcid.org/0000-0002-5375-3255

\section{REFERENCES}

1 Aira M, Hartikainen S, Sulkava R. Community prevalence of alcohol use and concomitant use of medication--a source of possible risk in the elderly aged 75 and older? Int J Geriatr Psychiatry 2005;20:680-5.

2 Dawson DA. Defining risk drinking. Alcohol Res Health 2011;34:144-56.

3 TCf SA. Substance abuse among older adults. treatment improvement protocol (tip) series, no. 26. Rockville (MD) - US: Substance Abuse and Mental Health Services Administration, 1998.

4 Blazer DG, Wu L-T. The epidemiology of at-risk and binge drinking among middle-aged and elderly community adults: national survey on drug use and health. Am J Psychiatry 2009;166:1162-9.

5 Graham K, Schmidt G. The effects of drinking on health of older adults. Am J Drug Alcohol Abuse 1998;24:465-81.

6 Blow FC, Walton MA, Barry KL, et al. The relationship between alcohol problems and health functioning of older adults in primary care settings. J Am Geriatr Soc 2000;48:769-74.

7 Cawthon PM, Fink HA, Barrett-Connor E, et al. Alcohol use, physical performance, and functional limitations in older men. J Am Geriatr Soc 2007;55:212-20.

8 Blanco C, Grant J, Petry NM, et al. Prevalence and correlates of shoplifting in the United States: results from the National epidemiologic survey on alcohol and related conditions (NESARC). Am J Psychiatry 2008;165:905-13.

9 Van Montfoort-De Rave KFG, De Weert-Van Oene GH, Beurmanjer $\mathrm{H}$, et al. Late-Onset alcohol dependence: patient-reported problems. Addict Res Theory 2017;25:139-45.

10 Adams WL, Cox NS. Epidemiology of problem drinking among elderly people. Int J Addict 1995;30:1693-716.

11 Pierucci-Lagha A. [Alcoholism and aging. 1. Epidemiology, clinical aspects and treatment]. Psychol Neuropsychiatr Vieil 2003;1:197-205.

$12 \mathrm{Hu}$ Y, Pikhart H, Malyutina S, et al. Alcohol consumption and physical functioning among middle-aged and older adults in central and eastern Europe: results from the HAPIEE study. Age Ageing 2015;44:84-9.

13 Tyas SL. Alcohol use and the risk of developing Alzheimer's disease. Alcohol Res Health 2001;25:299-306.

14 Volkert J, Schulz H, Härter M, et al. The prevalence of mental disorders in older people in Western countries - a meta-analysis. Ageing Res Rev 2013;12:339-53.

15 Woods AJ, Porges EC, Bryant VE, et al. Current heavy alcohol consumption is associated with greater cognitive impairment in older adults. Alcohol Clin Exp Res 2016;40:2435-44.

16 Ormstad H, Rosness TA, Bergem ALM, et al. Alcohol consumption in the elderly and risk of dementia related death--a Norwegian prospective study with a 17-year follow-up. Int $J$ Neurosci 2016;126:135-44.

17 Immonen S, Valvanne J, Pitkälä KH. The prevalence of potentia alcohol-drug interactions in older adults. Scand J Prim Health Care 2013;31:73-8.

18 Onder G, Landi F, Della Vedova C, et al. Moderate alcohol consumption and adverse drug reactions among older adults. Pharmacoepidemiol Drug Saf 2002;11:385-92.

19 Harford TC, Grant BF, Yi H-Y, et al. Patterns of DSM-IV alcohol abuse and dependence criteria among adolescents and adults: results from the 2001 national household survey on drug abuse. Alcohol Clin Exp Res 2005;29:810-28.
20 Boscarino JA, Moorman AC, Rupp LB, et al. Comparison of ICD-9 codes for depression and alcohol misuse to survey instruments suggests these codes should be used with caution. Dig Dis Sci 2017;62:2704-12.

21 Hajat S, Haines A, Bulpitt C, et al. Patterns and determinants of alcohol consumption in people aged 75 years and older: results from the MRC trial of assessment and management of older people in the community. Age Ageing 2004;33:170-7.

22 Ortolá R, García-Esquinas E, León-Muñoz LM, et al. Patterns of alcohol consumption and risk of frailty in community-dwelling older adults. J Gerontol A Biol Sci Med Sci 2016;71:251-8.

23 Hirata ES, Almeida OP, Funari RR, et al. Alcoholism in a geriatric outpatient clinic of São Paulo-Brazil. Int Psychogeriatr 1997;9:95-103.

24 Conigliaro J, Kraemer K, McNeil M. Screening and identification of older adults with alcohol problems in primary care. $J$ Geriatr Psychiatry Neurol 2000;13:106-14.

25 Di Bari M, Silvestrini G, Chiarlone M, et al. Features of excessive alcohol drinking in older adults distinctively captured by behavioral and biological screening instruments. An epidemiological study. $J$ Clin Epidemiol 2002;55:41-7.

26 Muñoz M, Ausín B, Santos-Olmo AB, et al. Alcohol use, abuse and dependence in an older European population: results from the MentDis_ICF65+ study. PLoS One 2018;13:e0196574.

27 Bosque-Prous M, Brugal MT, Lima KC, et al. Hazardous drinking in people aged 50 years or older: a cross-sectional picture of Europe, 2011-2013. Int J Geriatr Psychiatry 2017;32:817-28.

28 Nelson DE, Sattin RW, Langlois JA, et al. Alcohol as a risk factor for fall injury events among elderly persons living in the community. $J$ Am Geriatr Soc 1992;40:658-61.

29 Administration N. National Institute on Alcohol Abuse and Alcoholism.(2000). A Guide for Judges and Prosecutors, Washington, DC: Sentencing and Disposition of Youth DUI and Other Alcohol Offenses, 2006.

30 Grant BF, Chou SP, Saha TD, et al. Prevalence of 12-month alcoho use, high-risk drinking, and DSM-IV alcohol use disorder in the United States, 2001-2002 to 2012-2013: results from the National epidemiologic survey on alcohol and related conditions. JAMA Psychiatry 2017;74:911-23

31 Valderas JM, Starfield B, Sibbald B, et al. Defining comorbidity: implications for understanding health and health services. Ann Fam Med 2009;7:357-63.

32 Kessler RC, Berglund P, Demler O, et al. Lifetime prevalence and age-of-onset distributions of DSM-IV disorders in the National comorbidity survey replication. Arch Gen Psychiatry 2005;62:593-602.

33 Moher D, Liberati A, Tetzlaff J, et al. Preferred reporting items for systematic reviews and meta-analyses: the PRISMA statement. PLoS Med 2009;6:e1000097.

34 Zorzela L, Loke YK, loannidis JP, et al. PRISMA harms checklist: improving harms reporting in systematic reviews. $B M J$ 2016;352:i157.

35 Stroup DF, Berlin JA, Morton SC, et al. Meta-Analysis of observational studies in epidemiology: a proposal for reporting. meta-analysis of observational studies in epidemiology (moose) group. JAMA 2000;283:2008-12.

36 Green S, Higgins J. The Cochrane Handbook for systematic reviews of interventions. Chichester, UK: John Wiley \& Sons, Ltd, 2008

37 Sedgwick P. What is a non-randomised controlled trial? BMJ 2014;348:g4115.

38 Ferriter M, Huband N. Does the non-randomized controlled study have a place in the systematic review? A pilot study. Crim Behav Ment Health 2005;15:111-20.

39 WHO. Elderly population, 2019. Available: http://www.searo.who.int/ entity/health situation trends/data/chi/elderly-population/en/

40 Hasin DS, Stinson FS, Ogburn E, et al. Prevalence, correlates, disability, and comorbidity of DSM-IV alcohol abuse and dependence in the United States: results from the National epidemiologic survey on alcohol and related conditions. Arch Gen Psychiatry 2007:64:830-42.

41 Gunzerath L, Faden V, Zakhari S, et al. National Institute on alcohol abuse and alcoholism report on moderate drinking. Alcohol Clin Exp Res 2004;28:829-47.

42 Barnes AJ, Moore AA, Xu H, et al. Prevalence and correlates of at-risk drinking among older adults: the project share study. J Gen Intern Med 2010;25:840-6.

43 Novy J, Castelao E, Preisig M, et al. Psychiatric co-morbidities and cardiovascular risk factors in people with lifetime history of epilepsy of an urban community. Clin Neurol Neurosurg 2012;114:26-30.

44 Preisig M, Waeber G, Vollenweider P, et al. The PsyCoLaus study: methodology and characteristics of the sample of a population- 
based survey on psychiatric disorders and their association with genetic and cardiovascular risk factors. BMC Psychiatry 2009;9:9.

45 Firmann M, Mayor V, Vidal PM, et al. The CoLaus study: a population-based study to investigate the epidemiology and genetic determinants of cardiovascular risk factors and metabolic syndrome. BMC Cardiovasc Disord 2008;8:6.

46 Moher D, Liberati A, Tetzlaff J, et al. Preferred reporting items for systematic reviews and meta-analyses: the PRISMA statement. BMJ 2009;339:b2535.

47 Sterne JAC, Hernán MA, Reeves BC, et al. ROBINS-I: a tool for assessing risk of bias in non-randomised studies of interventions. BMJ 2016;355:i4919.

48 Higgins J, Green S. The Cochrane Handbook for systematic reviews of interventions. Chichester, UK: John Wiley \& Sons, Ltd, 2008.

49 Riley RD, Higgins JPT, Deeks JJ. Interpretation of random effects meta-analyses. BMJ 2011;342:d549.
50 Lau J, loannidis JPA, Terrin N, et al. The case of the misleading funnel plot. BMJ 2006;333:597-600.

51 Borenstein M, Hedges LV, Higgins JPT, et al. Prediction intervals: introduction to meta-analysis. UK: Chichester, 2009: 127-33.

52 Jones TV, Lindsey BA, Yount P, et al. Alcoholism screening questionnaires: are they valid in elderly medical outpatients? J Gen Intern Med 1993;8:674-8.

53 Suwała M, Gerstenkorn A. [Detection of alcohol problems among elderly people]. Psychiatr Pol 2007;41:703-13.

54 Krokstad S, Langhammer A, Hveem K, et al. Cohort profile: the HUNT study, Norway. Int J Epidemiol 2013;42:968-77.

55 Zeng Y. Towards deeper research and better policy for healthy aging - using the unique data of Chinese longitudinal healthy longevity survey. China Economic J 2012;5:131-49. 